\title{
ACHADOS ELETRENCEFALOGRÁFICOS EM 30 PACIENTES COM ILUSŌES DE MEMÓRIA
}

\author{
Rublins Moura Ribeiro *
}

As primeiras descrições de alteraçōes da memória na epilepsia temporal datam de 1898-1899, quando Hughlings Jackson descreveu o "dreamy state" 8, 9 . Êsses casos não são obrigatòriamente seguidos por convulsão focal ou generalizada; em geral, não há perda mas alteração da consciência. Em 1954, Penfield e Jasper 16 reuniram, sob o titulo de "ilusões da memória", as sensações de familiaridade ou fenômeno do já visto e as sensações de estranheza ou fenômeno do nunca visto. Esses autores obtiveram experimentalmente a reprodução da sensação de familiaridade ou de estranheza pela estimulação elétrica das faces lateral e superior do lobo temporal.

Não há mais dúvida quanto à importância do lobo temporal e estruturas a êle ligadas através do fórnix e formação hipocampal no mecanismo da memória, e, portanto, na evocação de experiências anteriormente vividas ${ }^{19}$. O córtex temporal é respon sável não sòmente pela gravação de experiências atuais, mas também pela interpretação de cada experiência vivida anteriormente, através da comparação dos fenômenos que dela participam e pela integração e armazenamento das impressões obtidas. Neste sistema diferenciado está integrada a formação descrita por Moruzzi e Magoun ${ }^{12}$ com o nome de sistema reticular e que tem a finalidade de manter as relações aferentes e eferentes do córtex cerebral. A importância que o núcleo ventral anterior do tálamo tem neste circuito é evidenciada pelas suas correlações com a ínsula, demonstradas pelas experiências em animais ${ }^{10}$ ou mediante observações anátomo-clínicas no homem ${ }^{2}$. Apesar dos testes de Rorscharsch e Tat não revelarem alterações significativas no psiquismo dos pacientes portadores de epilepsia temporal, quando comparados com outras formas de epilepsia ${ }^{11}$, Small e col. ${ }^{17}$ e Feindel e Penfield ${ }^{5}$ chamam a atenção para as perturbações mentais observadas nos pacientes com epilepsia temporal. Gibbs e Gibbs ${ }^{6}$ assinalam que mais da metade de seus pacientes com epilepsia temporal apresentam perturbações mentais.

\section{MATERIAL, MÉTODO E RESULTADOS}

Nosso material consta de 30 pacientes com epilepsia temporal que apresentaram llusões de memória: 20 eram do sexo feminino e 10 do sexo masculino; as idades variaram entre 10 e 40 anos. Os eletrencefalogramas foram registrados em

Trabalho da Clinica Neurológica da Fac. de Med. de Ribeirão Prêto, da Univi. de São Paulo, apresentado na I Reunião Anual da Academia Brasileira de Neurologia (Curitiba, iulho de 1963): * Assistente. 
aparelho Grass de 8 canais; a colocação dos eletrodos obedeceu à técnica do Montreal Neurological Institute.

Em todos os casos o exame eletrencefalográfico mostrou focos epilépticos ativos projetados no lobo temporal: 29 pacientes apresentavam foco unilateral e, um, foco de projeção bilateral. Dos 29 casos com foco unilateral 18 apresentavam projeção no Iobo temporal esquerdo e, 11, no lobo temporal direito (quadro 1).

\begin{tabular}{l} 
Lobo temporal esquerdo $\ldots \ldots \ldots \ldots \ldots \ldots \ldots \ldots \ldots$ \\
Lobo tempora! direito $\ldots \ldots \ldots \ldots \ldots \ldots \ldots \ldots \ldots \ldots \ldots$ \\
Repercussão bllateral sincrona $\ldots \ldots \ldots \ldots \ldots \ldots \ldots$ \\
\hline
\end{tabular}

Quadro 1 - Distribuição dos casos quanto à topografia.

Analisando a freqüência das manifestaçōes clinicas em relaçāo à localização do foro, verificamos que 21 pacientes apresentavam foco profundo e 9 tinham foco de situação superficial (quadro 2 ).

\begin{tabular}{|c|c|c|c|c|}
\hline \multirow{2}{*}{ Foco } & \multirow{2}{*}{ superficial } & \multirow{2}{*}{9 casos } & esquerdo & 5 \\
\hline & & & direjto & 4 \\
\hline \multirow{2}{*}{ Foco } & \multirow{2}{*}{ profundo } & \multirow{2}{*}{21 casos } & esquerdo & 13 \\
\hline & & & direito & 8 \\
\hline
\end{tabular}

A sensação de "estranheza" estêve presente 20 vêzes, sendo, em dois casos, manifestação isolada. A sensação de "já visto" foi verificada 6 vêzes, e a sensação de "estranheza" e "já visto" como manifestacão conjunta em 4 vêzes.

Ilusões de memória foram referidas por 9 pacientes (30\%) com focos de situacão cortical e 20 pacientes $(66,6 \%)$ com focos de situação profunda; em 17 pacientes $(56,6 \%)$ o foco estava situado no lobo temporal esquerdo, em 11 (36,6\%) no lobo temporal direito; em um $(3,3 \%)$ tinha repercussão bilateral. Portanto, em nosso material as ilusões de memória foram mais frequientes nos pacientes com focos de situaçāo subcortical no lobo temporal esquerdo.

Registramos também a presença de outras manifestações clínicas da epilepsia temporal que acompanhavam as ilusões de memória: crises psicomotoras em 24 casos; crises alucinatórias em 21 casos.

\section{COMENTARIOS}

Nossos 30 pacientes foram selecionados dentre 398 portadores de alteraçöes focais temporais no EEG. Assim, 7;5\% dos pacientes portadores de epilepsia temporal apresentavam ilusões de memória; esta percentagem è semelhante àquela ençontrada por Armbrust-Figueiredo ${ }^{1}$ quando estudou as manifestações clínicas de 1.000 pacientes portadores de focos temporais, dos quais $73(7 \%)$ apresentavam tais ilusões. 
Analisando a incidência das manifestações clínicas referidas pelos nossos pacientes, verificamos que a sensação de estranheza predominou, pois ocorreu em 20 pacientes $(66,6 \%$ dos casos); a sensação de familiaridade estêve presente em 6 pacientes $(20 \%)$ e a de estranheza e familiaridade como manifestações conjuntas em 4 pacientes $(13,3 \%)$. Como manifestações concomitantes encontramos crises do tipo automatismo psicomotor em 24 pacientes, e crises de alucinações em 21 pacientes. Esta alta incidência de automatismo psicomotor e ilusões de memória vem corroborar os trabalhos de Mulden e Daly em $1952^{13}$, que verificaram que as queixas mais usuais nos pacientes com epilepsia temporal eram do âmbito psiquiátrico; Weil em $1959^{20}$ mostrou lesões no lobo temporal de pacientes queixando-se de mêdo e de sensações irreais. Embora Gibbs e col. ${ }^{7}$ em 1948 encontrassem maior incidência de crises do tipo psicomotor em pacientes com foco temporal, as crises psicomotoras também podem depender de focos situados nos lobos frontal e parietal 4, pela repercussão que estas estruturas têm sôbre a região centrencefálica e lobo temporal ${ }^{3,15}$.

Discutindo as ilusões, Papez ${ }^{14}$ em 1937 elaborou as bases anatômicas para o substrato das emoções, e colocou sua sede no lobo temporal; em 1957 Stevens ${ }^{18}$ aventou a possibilidade de ser elaborado um homúnculo psíquico, de maneira semelhante ao homúnculo descrito na epilepsia de BravaisJackson.

\section{RESUMO}

Foram analisados do ponto de vista eletrencefalográfico 30 pacientes com "ilusões de memória": 29 pacientes apresentavam foco de projeção unilateral em um dos lobos temporais; um apresentava foco de projeção bilateral nos lobos temporais. Dos 29 casos com projeção unilateral, 18 apresentavam foco de projeção no lobo temporal esquerdo e 11 no lobo temporal direito. Em relação à situação do foco, 21 pacientes apresentavam foco profundo e 9 foco superficial.

\section{SUMMARY}

Electroencephalographic findings on 30 patients with illusions of memory.

Electroencephalographic studies were carried out on 30 patients with "illusions of memory": 29 had unilateral discharges in the temporal lobe and one had bilateral discharges in the temporal lobes. Eighteen patients showed focal discharges in the left temporal lobe and 11 in the right temporal lobe. In 21 patients the electroencephalogram showed subcortical discharges and in 9 cases focal cortical discharges.

REFERENCIAS

1. ARMBRUST-FIgUEIREDO, J. - Contribuição ao Estudo Clinico da Epilepsia Temporal. Tese de docência. Escola Paulista de Medicina, 1958. 2. ANGEVINE, J. B.; LOCKE, S.; YAKOVLEV, P. I. - Limbic nuclei of thalamus and con- 
nections of limbic cortex. Arch. Neurol., 7:518-528, 1962. 3. DELL, M. B. - L'epilepsie temporale. Presse Méd., 61:505-508, 1953. 4. FEGERSTEN, L.; ROGER, A. Frontal epileptogenic foci and their clinical correlations. Electroenceph. \& Clin. Neurophysiol., 13:905-913, 1961. 5. FEINDEL, W.; PENFIELD, W. — Localization of discharge in temporal lobe automatism. Arch. Neurol. \& Psychiat., 77:605-630, 1954. 6. GIBBS, F. A.; GIBBS, E. L. - Atlas of Electroencephalography, vol. II. Addison-Wesley, Cambridge (Mass.), 1952, págs. 169-170. 7. GIBBS, E. L.; GIBBS, F. A.; FUSTER, B. - Psychomotor epilepsy. Arch. Neurol. \& Psychiat., 60:331339, 1948. 8. JACKSON, J. H.; COLMAN, W. S. - Case of epilepsy with tasting movements and dreamy state. Very small patch of softening in the left uncinati gyrus. Brain, 21:580-590, 1898. In Taylor, J. - Selected Writings of John Hughlings Jackson, vol. 1: On Epilepsy and Epileptiform Convulsions. Hodder \& Stoughton, Londres, 1931, págs. 458-463. 9. JACKSON, J. H.; STEWART, J. P. — Epileptic attacks with a warning of a crude sensation of smell and with the intellectual aura (dreamy state) in a patient who had symptoms pointing to gross organic disease of the right temporo-sphenoidal lobe. Brain, 22:534-549, 1899. In Taylor, J. Selected Writings of John Hughlings Jackson, vol. 1: On Epilepsy and Epileptiform Convulsions. Hodder \& Stoughton, Londres, 1931, págs. 464-473. 10. LASHLEY, K. S. - Thalamo-cortical connections of the rat's brain. J. Comp. Neurol., 75:67-122, 1941. 11. MIRSKY, A. F.; PRIMAC, D. W.; AJMONE-MARSAN, C.; ROSVOLD, H. E.; STEVENS, J. R. - A comparison of the psychological test performance of patients with focal and nonfocal epilepsy. Exper. Neurol., 2:75-89, 1960. 12. MORUZZI, G.; MAGOUN, H. W. - Brain stem reticular formation and activation of the EEG. Electroenceph. \& Clin. Neurophysiol., 1:455-473, 1949. 13. MULDER, D. M.; DALY, D. - Psychiatric symptoms associated with lesions of temporal lobe. J.A.M.A., 150:173-176, 1952. 14. PAPEZ, J. W. - A proposed mechanism of emotion. Arch. Neurol. \& Psychiat., 38:725-743, 1947. 15. PENFIELD, W. - Epileptic automatism and the centroencephalic integrating system. Proc. Ass. Res. Nerv. \& Ment. Dis., 30:513-528, 1952. 16. PENFIELD, W.; JASPER, H. - Epilepsy and the Functional Anatomy of the Human Brain. Little Brown, Boston, 1954. 17. SMALL, J. G.; MILSTEIN, V.; STEVENS, J. R. - Are psychomotor epileptics different? Arch. Neurol., 7:187-194, 1962. 18. STEVENS, J. R. - The "march" of temporal lobe cpilepsy. Arch. Neurol. \& Psychiat., 77:227-236, 1957. 19. VICTOR, M.; ANGEVINE, V.; MANCALL, E. L.; FISHER, C. M. - Memoryloss with lesions of hippocampal formation. Arch. Neurol., 5:244-263, 1961. 20. WEIL, A. A. - Ictal emotions occurring in temporal lobe dysfunction. Arch. Neurol., 1:87-97, 1959.

Clínica Neurológica, Faculdade de Medicina - Ribeirão Prêto, São Paulo - Brasil. 